\title{
CÁLCULO DEL VALOR AGREGADO GENERADO POR LA UNIVERSIDAD DE NARIÑO EN RELACIÓN A LAS PRUEBAS SABER 11 - SABER PRO 2010-2014.
}

\author{
CALCULATION OF ADDED VALUE GENERATED BY THE UNIVERSIDAD DE \\ NARIÑO IN RELATION TO THE TESTS SABER 11 - SABER PRO 2010-2014.
}

\begin{abstract}
CÁLCULO DO VALOR ADICIONADO GERADO PELA UNIVERSIDAD DE NARIÑO EM RELAÇÃO AOS TESTS SABER 11 - SABRE PRO 2010-2014.
\end{abstract}

\section{BURGOS FLÓREZ _ Marco Antonio, RUALES SUÁREZ _ Katherin Julieth, BASTIDAS GARCÍA _ Yhony Estivel, ORTIZ BENAVIDES _ Edinson}

Mg. en Política Económica Internacional de la Universidad de Belgrano. Economista Universidad de Nariño. Docente tiempo completo Departamento de Economía Universidad de Nariño, Director Grupo de Investigación Coyuntura Económica y Social - CES. Email: marcoantonioburgos@gmail.com, Colombia.

Especialista en Finanzas, Economista de la Universidad de Nariño. Investigadora en Grupo de Investigación Coyuntura Económica y Social - CES. Email: katherin2320@hotmail.com, Colombia.

Economista de la Universidad de Nariño, Investigador en Grupo de Investigación Coyuntura Económica y Social - CES. Email: yhobas1991@gmail.com, Colombia.

Candidato a Doctor en Economía y Gestión Empresarial de la Universidad de Alcalá de Henares. Mg. en Economía de la Universidad de los Andes. Docente tiempo completo Departamento de 
Economía Universidad de Nariño, Investigador en Grupo de Investigación Coyuntura Económica y Social-CES. Email: edinsoneconomia@gmail.com, Colombia.

Recibido: 15 de agosto de 2019

Aprobado: 20 de diciembre

\section{DOI: https://doi.org/10.22267/rtend.192002.125}

\section{RESUMEN}

El presente artículo surge como resultado del proyecto de investigación con el mismo nombre realizado con financiación otorgada por la Universidad de Nariño a través del Sistema de Investigaciones en la Convocatoria Docente 2017, cuyo objetivo es la medición y análisis de los efectos generados por la Universidad de Nariño en los estudiantes que culminaron los estudios de pregrado en el periodo 2010-2014, mediante la estimación del valor agregado suscitado por la Universidad de Nariño a partir del cálculo de la diferencia entre los resultados de las Pruebas Saber 11 y Saber Pro. Este cálculo surge de la necesidad de evaluar el papel de la Universidad en la instrucción de sus estudiantes en las competencias básicas y ciudadanas. El enfoque de la investigación es cuantitativo y se utilizó información secundaria tanto de la Universidad como del Instituto Colombiano para la Evaluación de la Educación ICFES, encontrándose que la Universidad generó un valor agregado del $10 \%$ en el período estudiado.

Palabras clave: Calidad Educativa, Competencias Básicas, Examen de Estado, Institución de Educación Superior, Valor Agregado.

JEL: C02, I20, I21, I23, I29

\section{ABSTACT}

The present article that arises as a result of the research project with the same name made with funding granted by the Universidad de Nariño through the Research System in the 2017 Teaching Call, shows the measurement and analysis of the effects generated by the University of Nariño in the students who completed the undergraduate studies in the 2010-2014 period, by estimating the added value raised by the University of Nariño from the calculation of the 
difference between the results of the Saber 11 and Saber Pro Tests. It arises from the need to evaluate the role of the University in the instruction of its students in basic and citizen skills. The research approach is quantitative and secondary information from both the University and the Colombian Institute for the Evaluation of Education ICFES, finding that the University generated an added value of $10 \%$ in the period studied.

Keywords: Educational Quality, Basic Competences, State Exam, Institution of Higher Education, Added Value.

JEL: C02, I20, I21, I23, I29

\section{RESUMO}

O presente artigo, resultado de um projeto de pesquisa com o mesmo nome realizado com financiamento concedido pela Universidad de Nariño através do Sistema de Pesquisa na Chamada para Ensino de 2017, mostra a medição e a análise dos efeitos gerados pela Universidade de Nariño nos alunos que concluíram os estudos de graduação no período 20102014, estimando o valor agregado gerado pela Universidade de Nariño a partir do cálculo da diferença entre os resultados dos testes Sabre 11 e Sabre Pro. Precisa avaliar o papel da Universidade na instrução de seus alunos nas habilidades básicas e cidadãs. A abordagem da pesquisa é quantitativa e informações secundárias da Universidade e do Instituto Colombiano de Avaliação da Educação do ICFES, constatando que a Universidade gerou um valor agregado de $10 \%$ no período estudado.

Palavras-Chave: Qualidade educacional, competências básicas, Exame Estadual, Instituição de Ensino Superior, Valor Adicionado.

JEL: C02, I20, I21, I23, I29 
Cálculo del valor agregado generado por la Universidad de Nariño en relación a las pruebas saber 11 - saber pro 2010-2014.

\section{INTRODUCCIÓN}

A medida que el proceso de la internacionalización de talento humano y la globalización de ciencia y tecnología se incrementan, la necesidad de mejorar las habilidades, aptitudes, conocimientos y destrezas de los individuos se vuelve cada vez más imperioso, y con ello, el menester de mejorar la calidad de la educación, especialmente en países en vías de desarrollo como Colombia, va de la mano con la exigencia de mayor productividad y competitividad para superar los grandes retos de integración a la modernidad, crecimiento y desarrollo sostenible y sustentable.

De esa manera, es preciso mencionar que a lo largo y ancho del mundo, el mayor determinante del progreso de los países es el nivel de formación con el que cuentan los seres humanos, su calidad, constancia y disciplina y de los procesos investigativos derivados, una tarea que además de estar en las manos de la unidad familiar, corresponde a los centros educativos, del menor al mayor grado de aprendizaje, y entre los cuales, los centros de educación superior, tienen una tarea fundamental que será estudiada a lo largo del proceso investigativo.

Este trabajo se propone medir y analizar cuáles son los efectos derivados de la educación superior obtenida en la Universidad de Nariño por los estudiantes de las diversas carreras ofertadas en cuanto a su formación en competencias básicas, para lo cual se tomaron los resultados de dos pruebas establecidas por el Ministerio de Educación Nacional: en primera instancia, SABER 11 o el mecanismo por el cual los estudiantes que culminan su formación media pueden acceder a estudios superiores y en segunda instancia, SABER PRO o el requisito imperativo para poder culminar dichos estudios y poder desempeñarse como un profesional, todo con el objetivo de determinar qué tan bien o mal lo está haciendo la Universidad, y cuáles son las estrategias que permiten establecer un mejoramiento de la calidad de la educación.

La importancia de realizar este tipo de estudios surgió de la necesidad de evaluar el papel de la Universidad en la instrucción de todos y cada uno de sus estudiantes, no solo en materias que confieren a la especificidad de cada carrera profesional, sino en las competencias básicas y ciudadanas que le otorgan al universitario cimientos necesarios para poder desarrollar su conocimiento de manera integral. Así pues, este proyecto de investigación se encaminó, 
esencialmente, a la medición y análisis del valor agregado que hace la Universidad de Nariño sobre los estudiantes adscritos a los distintos programas de pregrado, investigación que se considera novedosa en tanto no se cuenta con antecedentes que permitan establecer un estado del arte riguroso y por ende, un análisis longitudinal del progreso de la Universidad en este escenario.

\section{REFERENTES TEÓRICO - CONCEPTUALES}

\section{Antecedentes}

En el ámbito nacional, no es frecuente encontrar estudios encaminados a investigar cuál es el valor agregado que las instituciones de educación superior adicionan en la formación de los individuos. Esta falta de trabajos al respecto se puede atribuir a la dificultad que implica el seguimiento a estudiantes que ingresan al sistema educativo superior o a la diversidad de las pruebas que miden el aprendizaje, y que impiden comparar los resultados.

Sin embargo, el Instituto Colombiano para el Fomento de la Educación Superior (ICFES), ha avanzado en este campo, permitiendo que las pruebas aplicadas de manera periódica, tengan un componente similar y comparable en los diferentes niveles de educación; de esta manera, Colombia es pionero en este tipo de investigaciones, ya que es de los pocos países donde los estudiantes deben presentar una evaluación de aprendizajes al final de sus ciclos educativos.

En su estudio "Medición de los efectos de la educación superior en Colombia sobre el aprendizaje estudiantil", el ICFES utiliza modelos de Valor Agregado, los cuales intentan, mediante procedimientos estadísticos, evaluar la eficiencia educativa, haciendo énfasis en el progreso que los estudiantes presentan en el tiempo (Instituto Colombiano para el Fomento de la Educación Superior, 2014).

Isáziga \& Gabalá (2014), tambien utilizan la metodología de valor agregado para hacer un estudio en referencia al aporte de la educación superior al aprendizaje, utilizando datos obtenidos por un grupo de personas en las pruebas SABER 11, como indicador de las condiciones de entrada de los estudiantes antes de comenzar el proceso de formación universitario; y los resultados de los mismos individuos en la prueba SABER PRO, como indicador de las 
condiciones de salida del proceso. La investigación se realiza por medio de una regionalización de los departamentos basados en la metodología CORPES del pais, debido a que zonas del territorio comparten ciertas características que permiten hacer la sectorización y obtener mejores resultados.

El análisis se hace en el modulo de "Comprensión Lectora" y se concluye que hay un aporte de las instituciones en el mejoramiento del aprendizaje de los individuos, haciendo una comparación entre los resultados en las pruebas SABER 11 y SABER PRO.

Para la regíon, no existen investigaciones de este tipo, por lo cual este trabajo se torna muy relevante, teniendo en cuenta que la Universidad de Nariño, es un gran absorvente de población estudiantil que ingresa a la educación superior en el suroccidente colombiano. Así mismo, se puede establecer si esta institución genera un aporte académico en la formación de profesionales, que van a aportar en el desarrollo regional, como la misión de la universidad lo describe.

\section{Marco Teórico}

Desde el año 2008, el Ministerio de Educación Nacional (2011) emprendió acciones tendientes a la formulación de competencias genéricas, o transversales a todos los núcleos de formación en educación superior, con el fin de monitorear la calidad de la educación superior en el país. Según Clavijo (2008), la propuesta de centrarse en las "competencias" ha venido ganando fuerza en el campo de la evaluación educativa, como alternativa a las tradicionales evaluaciones de "rendimiento académico". La idea central es desplazar la evaluación del manejo de contenidos curriculares al desarrollo de conocimientos, valores, actitudes, habilidades, en diferentes contextos y con sentido.

En tal sentido, con el fin de contribuir al mejoramiento de la calidad de la educación y determinar los factores que inciden en ella, a través de información pertinente y oportuna, el Instituto Colombiano para la Evaluación de la Educación - ICFES, apoya al Ministerio de Educación Nacional en la realización de los exámenes de Estado, orientados a la evaluación de competencias, con el fin de evaluar la educación en todos sus niveles (Instituto Colombiano para el Fomento de la Educación Superior, 2018). 
Cálculo del valor agregado generado por la Universidad de Nariño en relación a las pruebas saber 11 - saber pro 2010-2014.

Los exámenes de calidad de la educación evalúan en los términos establecidos por el estado la calidad que tienen los estudiantes del bachillerato y de la universidad, posibilitando mirar el papel de las instituciones y el desempeño del estudiante, siendo la Prueba Saber 11, aquella que posibilita generar una exigencia de las mismas para las instituciones y la validación de la calidad del estudiante para continuar con sus estudios superiores y acceder a los beneficios de la educación pública y los estímulos del estado en torno a la ampliación de la cobertura educativa superior. Por su parte, la Prueba Saber Pro, pretende evaluar las competencias del profesional con miras al ámbito laboral y postgradual, así como los beneficios que tiene el estado para los profesionales sobresalientes, en donde también se evaluara en parte la labor educativa de la Institución de educación superior IES, siendo este un aspecto importante en la evaluación de la calidad de la educación superior en la renovación del Registro calificado del programa o su Acreditación de alta calidad.

De este modo, resultan interesantes los modelos estadísticos de Valor agregado, los cuales buscan cuantificar cuánto aportan las instituciones educativas a las competencias de sus estudiantes, independiente de las condiciones de entrada de los alumnos.

Cuando un alumno entra a un centro educativo llega con unas habilidades previas, por lo que sus capacidades al terminar su ciclo académico no se deben solo a lo que aprendió por su paso en él, sino también a lo que sabían antes de entrar al mismo (Instituto Colombiano para el Fomento de la Educación Superior, 2018).

En el ámbito internacional, no es frecuente encontrar estudios encaminados a investigar cuál es el valor agregado que las instituciones de educación superior adicionan en la formación de los individuos, Colombia es pionero en este tipo de investigaciones, y es de los pocos países donde los estudiantes deben presentar una evaluación de aprendizaje al final de sus ciclos educativos. Además, todos los exámenes de Estado y de educación básica que aplica el ICFES están estandarizados, en la medida en que las condiciones de aplicación y el procesamiento de los resultados son uniformes.

Entre algunas investigaciones relacionadas con el tema de estudio, se encuentra la "Medición de los efectos de la educación superior en Colombia sobre el aprendizaje estudiantil" (Instituto 
Colombiano para el Fomento de la Educación Superior, 2014), en donde se utiliza modelos de Valor Agregado, los cuales intentan, mediante procedimientos estadísticos, evaluar la eficiencia educativa, haciendo enfasis en el progreso que los estudiantes presentan en el tiempo. La inferencia causal es la base del procedimiento metodológico de esta investigación.

Por lo tanto, el principal propósito de esta investigación es medir y analizar el valor agregado generado por la Universidad de Nariño en relación a las pruebas Saber 11 y Saber Pro durante el periodo 2010-2014, y su incidencia en la calidad educativa de la institución.

Ahora bien, la visión de la educación como una garantía futura para el mejoramiento de la calidad de vida del individuo y su familia, ha hecho que crezca una cultura encaminada a conseguir un mayor grado de formación por las generaciones más jóvenes, y es por ello que la política pública en los países del mundo, particularmente en las naciones en vía de desarrollo, ha empezado a ver a la educación como un pilar fundamental en el cierre progresivo de las brechas de desarrollo, respecto a las potencias mundiales.

Cuando el mundo empieza a hablar de desarrollo después de la segunda guerra mundial, las lecturas de la educación como un elemento clave en el avance de los países a mejores condiciones de vida, se convierte en un importante tema de estudio que conlleva la idea de que los procesos formativos deben permitir que no solo el país le aporte a sus ciudadanos, sino que cada una de las personas, desde su formación, sea una célula de desarrollo, contribuyendo de manera efectiva a la generación de diferentes valores a la sociedad en su conjunto. Al ser el desarrollo una temática tan compleja como las diferentes teorías que se han propuesto al respecto, la educación aparece en cada una de ellas con un tinte propio en relación al papel que juega en los cambios conocidos como desarrollistas.

Desde los autores clásicos del pensamiento económico y los que le precedieron en la escuela neoclásica, se ha visto la educación como un factor importante en relación a la producción económica, teniendo en cuenta que la formación adquirida, ya sea en la academia o a través de la experiencia, propician un desempeño más adecuado en las labores a las cuales se dedican las personas, ganando con el tiempo mayor experticia en el trabajo y generando una mayor productividad. 
Cálculo del valor agregado generado por la Universidad de Nariño en relación a las pruebas saber 11 - saber pro 2010-2014. BURGOS FLÓREZ _ Marco Antonio, RUALES SUÁREZ _ Katherin Julieth, BASTIDAS GARCÍA _ Yhony Estivel, ORTIZ

De acuerdo a Martínez (1997) citado en Cardona, et al. (2007), este interés desembocó en el surgimiento y desarrollo de la teoría del capital humano, consolidando la economía de la educación. Más adelante, autores como Solow (1950), Schultz (1961), Becker (1964) y Mincer (1974) (citados por Cardona, et al., (2007)), contribuyeron al desarrollo de la teoría del Capital Humano que nace como una rama de la ciencia económica; a partir de esto, se ve la educación no como un gasto, sino como una inversión que incrementa la productividad en el largo plazo; pero además, genera la existencia de un tercer factor de producción después del capital y el trabajo, que hace referencia al avance técnico y tecnológico en los procesos productivos.

Con esto, el trabajo como fuerza productiva se visualiza desde dos puntos de vista; por una parte, las actividades repetitivas donde se usa principalmente la fuerza, una habilidad desarrollada por la repetición de actividades o procedimientos mecánicos que conllevan la responsabilidad de un puesto de trabajo; y por otra parte, el desarrollo del conocimiento aplicado a una mejor manera de realizar ciertas actividades; este último reviste lo que se conoce como la innovación y emprendimiento, entre otras habilidades no tangibles que se desarrollan con la formación académica alineada con las aptitudes de las personas.

Hacia finales del siglo XX y comienzos del XXI, diferentes autores han teorizado sobre visiones menos economicistas del desarrollo, contemplando diferentes esferas del conocimiento y de la acción social, lo que resulta en una visión multidimensional del desarrollo. Amartya Sen gana el premio nobel de Economía en el año 2008 por sus aportes al estudio del desarrollo con una teoría que se aleja de las convencionales y ortodoxas.

Sen (1997) introduce el concepto de Capacidad Humana, en contraste al de Capital Humano, cuya principal diferencia radica en que el primero pone mayor énfasis en el rol del ser humano como productor de bienes, mientras que el segundo (Propuesto por Sen), centra su atención en la capacidad de los individuos para vivir la vida, y por ello se analizan los motivos que éstos poseen para valorar y aumentar las alternativas reales entre las cuales poder optar. Estas capacidades también son llamadas libertades, en tanto le permiten al individuo conseguir los requerimientos para el disfrutar pleno de su vida, de donde se retoma el desarrollo como libertad (Sen, 1997). 
Cálculo del valor agregado generado por la Universidad de Nariño en relación a las pruebas saber 11 - saber pro 2010-2014.

BURGOS FLÓREZ _ Marco Antonio, RUALES SUÁREZ _ Katherin Julieth, BASTIDAS GARCÍA _ Yhony Estivel, ORTIZ

BENAVIDES _ Edinson

Desde esta teoría, la educación es una capacidad esencial, que les permite a las personas acceder a mejores escenarios y calidad de vida, no solamente desde el punto de vista financiero, sino desde la satisfacción misma del saber hacer.

Desde las dos teorías expuestas, la educación tiene un papel importante en el desarrollo económico y social de los individuos y de las regiones, contribuyendo a un mejor escenario en cuanto a calidad de vida, de tal manera que los procesos educativos van a tener una consecuencia directa en diferentes esferas, que demandan una continua evaluación de dichos procesos que implican una inversión relativamente importante en el gasto público, así como en los desembolsos individuales que las personas realizan en la búsqueda de una formación de mayor calidad.

Un estudio como el presente, entonces, contribuye a realizar una evaluación minuciosa de si las universidades están o no cumpliendo con su papel de educadoras y formadoras de capital humano, y una forma de medirlas es a través del análisis del valor agregado que generan en los estudiantes con respecto a su formación en competencias genéricas.

\section{METODOLOGÍA}

Para desarrollar la presente investigación, se trabajó con toda la población estudiantil de la Universidad de Nariño que haya recibido su título profesional entre el semestre A de 2010 y el Semestre A de 2014. Toda la información fue obtenida desde fuentes secundarias y se le dio el tratamiento requerido.

Aunque el ICFES no ha logrado una estandarización de las pruebas Saber 11 y Saber Pro, está adelantando procesos que permitan una comparabilidad de estas pruebas en el futuro, de tal manera que se puedan llevar a cabo estudios de análisis vertical de los resultados. Por el momento, se pueden establecer diferentes herramientas estadísticas que permitan unos resultados que expliquen en mayor medida el valor agregado de la Institución a partir de los datos existentes. Para lograr el objetivo planteado, se recurrió a fuentes secundarias como documentos, tesis, libros y datos proporcionados por el ICFES, así como también a información obtenida de diversas fuentes electrónicas referentes al tema en cuestión y se llevó a cabo una investigación aplicada con la utilización de relaciones causales. 


\section{RESULTADOS Y DISCUSIÓN}

Según el ICFES (2014, pág. 5), los modelos de Valor Agregado son modelos de carácter estadístico que intentan evaluar la efectividad educativa haciendo énfasis en el progreso de los estudiantes en el tiempo y para lograrlo, en Colombia se utilizan los puntajes actuales de Saber Pro en comparación con los puntajes predichos a partir de los resultados observados en Saber $11^{\circ}$, para con ello evaluar el desempeño de las instituciones. Por tanto, se procedió a realizar un modelo que analiza los resultados obtenidos por los estudiantes de la Universidad de Nariño en las Pruebas Saber $11^{\circ}$ como línea de base y las Pruebas Saber Pro como variables de salida, para con ello poder analizar el impacto generado en los estudiantes objeto de estudio y en la institución en general.

En primera instancia se optó por conocer la estructura de cada una de las pruebas a analizar, teniendo en cuenta que tanto las Pruebas Saber $11^{\circ}$ como las Pruebas Saber Pro presentaron modificaciones en su metodología y los puntajes desde 2012 a 2015 y 2016 para la Prueba Saber Pro no son comparables, como tampoco lo son los puntajes entre los periodos 2006 a 2014_1 y 2014_2 a 2017_2 de la Prueba Saber 11 ${ }^{\circ}$. Por lo anterior, se procedieron a analizar datos hasta 2014_1 y las estructuras de las pruebas que se estudiaron fueron las vigentes en dichos periodos.

PRUEBAS SABER 11º: La estructura del examen hasta el primer semestre de 2014 constó de un núcleo común, con ocho pruebas de carácter obligatorio y un componente flexible que otorgaba la posibilidad de elegir entre una prueba de profundización o una interdisciplinar, teniendo en cuenta las preferencias de cada examinando. Dicha estructura se resume en la tabla 1 .

\section{Tabla 1}

Estructura de la Prueba Saber 11, 2014

Núcleos

Núcleo Común

Componente Flexible

\section{Pruebas (Hasta el primer semestre de 2014)}

Lenguaje, Matemáticas, Biología, Física, Química, Ciencias Sociales, Filosofía, Inglés

Profundización en alguna de las siguientes áreas: Biología, Ciencias Sociales, Lenguaje o Matemáticas, o Física, Química 
Interdisciplinar Violencia y Sociedad o Medio Ambiente, Filosofía, Inglés

Fuente: ICFES (2014)

PRUEBA SABER PRO: A partir del año 2010, el ICFES incluyó "la evaluación de competencias genéricas, entendidas como aquellas que todos los estudiantes deben desarrollar independiente del énfasis de formación, y la evaluación de competencias comunes a grupos de programas con características de formación similares" (Instituto Colombiano para el Fomento de la Educación Superior, 2015). Con respecto a la evaluación de competencias genéricas, la estructura del examen se aprecia en la tabla 2.

\section{Tabla 1}

Estructura de la Prueba Saber Pro, 2014

\begin{tabular}{llc}
\hline \multicolumn{1}{c}{ Sesión } & \multicolumn{1}{c}{ Módulo } & Preguntas por Módulo \\
\hline Primera sesión & Lectura Crítica & 35 \\
Competencias genéricas & Razonamiento Cuantitativo & 35 \\
& Competencias Ciudadanas & 35 \\
& Comunicación Escrita & 1 \\
& Inglés & 45 \\
\hline
\end{tabular}

Fuente: ICFES (2017)

"La educación en Colombia, en todos sus niveles, está orientada hacia el desarrollo de competencias y, en particular, las competencias genéricas están presentes en todos los niveles de formación" (Instituto Colombiano para el Fomento de la Educación Superior, 2013, pág. 23) y todas las evaluaciones están estructuradas en dicho eje, es decir las Pruebas Saber $3^{\circ}, 5^{\circ}, 9^{\circ}$ y Pro. No obstante, a pesar de que el examen Saber $11^{\circ}$ evalúa competencias genéricas, no estaba diseñado siguiendo ese eje.

Entre las modificaciones propuestas por el ICFES (2013, págs. 27-28) en la estructura del examen, se encuentran las siguientes: 
Cálculo del valor agregado generado por la Universidad de Nariño en relación a las pruebas saber 11 - saber pro 2010-2014.

BURGOS FLÓREZ _ Marco Antonio, RUALES SUÁREZ _ Katherin Julieth, BASTIDAS GARCÍA _ Yhony Estivel, ORTIZ

BENAVIDES _ Edinson

- Fusionar las pruebas de Lenguaje y de Filosofía en una prueba de Lectura Crítica.

- Fusionar las pruebas de Física, de Química y de Biología en una prueba de Ciencias.

- Incluir el área de Ciencia, Tecnología y Sociedad en la nueva prueba de Ciencias, y responder así a lo que se encuentra establecido en los Estándares.

- Reemplazar la prueba de Ciencias Sociales por una que incluya la evaluación de Competencias Ciudadanas: Sociales y Ciudadanas

- Distinguir en la prueba de Matemáticas entre aquello que es genérico (el razonamiento cuantitativo) y aquello que no lo es.

Es posible manifestar, entonces, que aun cuando se trabajarán resultados anteriores a 2014, la equivalencia propuesta entre componentes de Saber $11^{\circ}$ y Saber Pro es la que se observa en la tabla 3 debido que son exámenes que comparten sus características y son estandarizadas por el ICFES.

\section{Tabla 2}

Comparabilidad de los módulos genéricos entre saber 11 y saber pro

\begin{tabular}{ll}
\hline Saber $\mathbf{1 1}^{\circ}$ antes del $\mathbf{2 0 1 4}$ & Saber Pro \\
\hline Matemáticas & Razonamiento Cuantitativo \\
Lenguaje y Filosofía & Lectura Crítica \\
Ciencias Sociales & Competencias Ciudadanas \\
Inglés & Inglés
\end{tabular}

Física, Química Y Biología

Comunicación escrita

Fuente: ICFES (2013) Elaboración: Esta investigación

Las áreas de física, química y biología (ciencias naturales a partir de 2014), evaluadas en Saber $11^{\circ}$ antes de 2014 y la de comunicación escrita, evaluada en Saber Pro, no se tendrán en cuenta debido a la ausencia de su equivalente.

Las cuatro áreas examinadas en esta investigación se consideran transversales a la formación de los profesionales en cualquier área de conocimiento, tomando en cuenta que una de ellas 
tendrá más intensidad por la naturaleza de cada plan de estudios. Respetando las metodologías utilizadas desde el 2010 hasta el 2014, que permiten la comparabilidad de las áreas genéricas evaluadas en Saber 11 y Saber Pro, en este estudio se aplica la metodología de medición de valor agregado propuesta por Monroy, Aguirre, \& Espitia (2018), la cual se resume en tres pasos:

1. Realizar un promedio simple de los puntajes de los componentes que se van a tener en cuenta y que según la nueva estructura contienen más de una prueba, como es el caso de: Sociales y Competencias Ciudadanas y Lectura Crítica.

2. Ahora que se posee un solo resultado por componente, cada uno de estos son divididos entre el valor máximo del puntaje respectivo, para hallar el porcentaje de desempeño de los estudiantes frente a cada prueba.

3. Finalmente se calcula la diferencia entre el porcentaje de desempeño de cada uno de los componentes entre la prueba Saber 11 y la prueba Saber Pro para cada uno de los estudiantes, determinando así el valor agregado que aportó la universidad de manera individual.

A partir de este cálculo no complejo, se llega a un porcentaje que representa el valor ganado por los estudiantes que cursaron un plan de estudios universitario medido a través de las pruebas estatales Saber Pro, respecto a su aprendizaje en la educación media medida con la prueba de estado Saber 11.

Se encontró que la Universidad de Nariño genera en promedio un $10 \%$ de valor agregado a sus estudiantes durante el periodo estudiado, no obstante, el valor agregado es atribuido por cada componente como se observa en la tabla 4, en donde es el módulo de inglés el que más aporta, ubicándose en un $13,50 \%$, seguido de razonamiento cuantitativo con $13,37 \%$.

\section{Tabla 3}

Medidas de centralización y dispersión del valor agregado medido por componentes. Universidad de Nariño, 2010 - 2014

Estadísticos Lectura Razonamiento Competencias Inglés


Cálculo del valor agregado generado por la Universidad de Nariño en relación a las pruebas saber 11 - saber pro 2010 -2014. BURGOS FLÓREZ _ Marco Antonio, RUALES SUÁREZ _ Katherin Julieth, BASTIDAS GARCÍA _ Yhony Estivel, ORTIZ

\begin{tabular}{ccccc}
\hline & Crítica & Cuantitativo & Ciudadanas & \\
\hline Media & $6,59 \%$ & $13,37 \%$ & $2,89 \%$ & $13,50 \%$ \\
Moda & $9,53 \%$ & $12,52 \%$ & $8,30 \%$ & $18,26 \%$ \\
Varianza & 0,008 & 0,009 & 0,008 & 0,008 \\
Desviación & 0,090 & 0,094 & 0,087 & 0,087 \\
Mínimo & $-20 \%$ & $-17 \%$ & $-27 \%$ & $-35 \%$ \\
Máximo & $36 \%$ & $40 \%$ & $28 \%$ & $38 \%$ \\
\hline
\end{tabular}

Fuente: Esta investigación

El componente en el que menos les aportó la Universidad a sus estudiantes es en competencias ciudadanas con un $2,89 \%$. En la competencia de razonamiento cuantitativo se hace posible observar que se obtuvo un máximo de $40 \%$ en valor agregado generado por la universidad, mientras que en los mínimos, el que más resalta es el obtenido en inglés con un $35 \%$.

En la tabla 5 se observa el valor agregado generado por la Universidad de Nariño en las competencias a lo largo de los años, cuyas cifras encontradas permiten afirmar que hay grandes fluctuaciones en el periodo.

\section{Tabla 4}

Media valor agregado por año y por competencia. Universidad de Nariño, 2010 - 2014.

\begin{tabular}{ccccc}
\hline Año & $\begin{array}{c}\text { Lectura } \\
\text { Crítica }\end{array}$ & $\begin{array}{c}\text { Razonamiento } \\
\text { Cuantitativo }\end{array}$ & $\begin{array}{c}\text { Competencias } \\
\text { Ciudadanas }\end{array}$ & Inglés \\
\hline 2010 & $4,75 \%$ & $13,28 \%$ & $2,22 \%$ & $12,68 \%$ \\
2011 & $13,64 \%$ & $12,33 \%$ & $4,06 \%$ & $16,47 \%$ \\
2012 & $9,78 \%$ & $13,79 \%$ & $7,11 \%$ & $16,35 \%$ \\
2013 & $6,98 \%$ & $21,51 \%$ & $6,94 \%$ & $11,82 \%$ \\
2014 & $7,66 \%$ & $16,55 \%$ & $1,53 \%$ & $12,51 \%$ \\
\hline
\end{tabular}

Fuente: Esta investigación 
En general, el valor agregado por la Universidad de Nariño muestra una tendencia ascendente, permitiendo afirmar que conforme pasa el tiempo, el papel de la Universidad en la formación de sus estudiantes en cuanto a competencias genéricas se refiere, ha ido mejorando, contribuyendo al mejoramiento de la calidad de la educación y a la formación de capital humano, tal como lo menciona el Ministerio de Educación Nacional (2018) y se exterioriza en los modelos de Solow, Schultz, entre otros, en donde se ve a la educación no como un gasto sino como una inversión que además de permitir el avance técnico y tecnológico, posibilita el incremento de la productividad en los procesos que el individuo desarrolla.

En el análisis del valor agregado por programas académicos, los mayores puntajes de agregación de valor se obtienen en los módulos de Razonamiento Cuantitativo e Inglés; mientras que los módulos con menores porcentajes de valor agregado son lectura crítica y competencias ciudadanas; en estos últimos, se generaron resultados negativos para algunos programas, lo que implica menores resultados para estas áreas por parte de los estudiantes en las pruebas Saber Pro en relación a las obtenidas en Saber 11 (ver tabla 6).

Esto se debe entrar a evaluar de manera particular, teniendo en cuenta otras metodologías y datos adicionales que resulten en mayor claridad en relación al aporte que hace el plan de estudios en el conocimiento de los estudiantes que lo cursan, y que por ende, sobrepasan los límites de esta investigación.

\section{Tabla 6}

Valor agregado por programa de pregrado de la Universidad de Nariño. 2010 - 2014

\begin{tabular}{lccccc}
\hline $\begin{array}{c}\text { Programas De } \\
\text { Pregrado }\end{array}$ & $\begin{array}{c}\text { Lectura } \\
\text { Crítica }\end{array}$ & $\begin{array}{c}\text { Razonamiento } \\
\text { Cuantitativo }\end{array}$ & $\begin{array}{c}\text { Competencias } \\
\text { Ciudadanas }\end{array}$ & Inglés & Total \\
\hline ADMINISTRACIÓN DE & $7,39 \%$ & $9,66 \%$ & $5,79 \%$ & $16,41 \%$ & $9,81 \%$ \\
EMPRESAS & $9,03 \%$ & $9,44 \%$ & $5,53 \%$ & $8,74 \%$ & $8,19 \%$ \\
ARQUITECTURA & $5,28 \%$ & $16,25 \%$ & $6,49 \%$ & $10,10 \%$ & $9,53 \%$ \\
ARTES VISUALES & $8,60 \%$ & $5,12 \%$ & $2,14 \%$ & $12,12 \%$ & $6,99 \%$ \\
BIOLOGÍA & & & & & \\
\end{tabular}


Cálculo del valor agregado generado por la Universidad de Nariño en relación a las pruebas saber 11 - saber pro 2010 -2014. BURGOS FLÓREZ _ Marco Antonio, RUALES SUÁREZ _ Katherin Julieth, BASTIDAS GARCÍA_ Yhony Estivel, ORTIZ BENAVIDES _ Edinson

\begin{tabular}{|c|c|c|c|c|c|}
\hline COMERCIO & $3,29 \%$ & $23,05 \%$ & $6,97 \%$ & $11,22 \%$ & $11,13 \%$ \\
\hline \multicolumn{6}{|l|}{ INTERNACIONAL Y } \\
\hline \multicolumn{6}{|l|}{ MERCADEO } \\
\hline DERECHO & $6,05 \%$ & $14,48 \%$ & $5,04 \%$ & $12,05 \%$ & $9,41 \%$ \\
\hline DISEÑO GRÁFICO Y & $5,68 \%$ & $18,64 \%$ & $6,07 \%$ & $13,55 \%$ & $10,98 \%$ \\
\hline \multicolumn{6}{|l|}{ MULTIMEDIAL } \\
\hline DISEÑO INDUSTRIAL & $8,52 \%$ & $13,61 \%$ & $6,13 \%$ & $14,53 \%$ & $10,70 \%$ \\
\hline ECONOMÍA & $4,33 \%$ & $10,82 \%$ & $-2,25 \%$ & $11,67 \%$ & $6,14 \%$ \\
\hline FÍSICA & $-8,39 \%$ & $11,29 \%$ & $-3,35 \%$ & $4,78 \%$ & $1,08 \%$ \\
\hline ING. AGROFORESTAL & $5,51 \%$ & $13,48 \%$ & $0,31 \%$ & $16,43 \%$ & $8,93 \%$ \\
\hline ING. & $8,55 \%$ & $12,71 \%$ & $2,56 \%$ & $16,48 \%$ & $10,08 \%$ \\
\hline \multicolumn{6}{|l|}{ AGROINDUSTRIAL } \\
\hline ING. AGRONÓMICA & $2,11 \%$ & $16,02 \%$ & $4,67 \%$ & $15,33 \%$ & $9,53 \%$ \\
\hline ING. AMBIENTAL & $9,80 \%$ & $9,48 \%$ & $5,34 \%$ & $10,06 \%$ & $8,67 \%$ \\
\hline ING. CIVIL & $1,71 \%$ & $6,88 \%$ & $-5,28 \%$ & $9,04 \%$ & $3,09 \%$ \\
\hline ING. DE SISTEMAS & $9,34 \%$ & $15,36 \%$ & $0,59 \%$ & $10,08 \%$ & $8,84 \%$ \\
\hline ING. ELECTRÓNICA & $-5,99 \%$ & $0,74 \%$ & $-2,03 \%$ & $1,51 \%$ & $-1,44 \%$ \\
\hline ING. EN PRODUCCIÓN & $16,95 \%$ & $13,09 \%$ & $7,44 \%$ & $14,63 \%$ & $13,03 \%$ \\
\hline \multicolumn{6}{|l|}{ ACUÍCOLA } \\
\hline LIC. EN INGLES Y & $3,20 \%$ & $14,91 \%$ & $3,33 \%$ & $13,40 \%$ & $8,71 \%$ \\
\hline \multicolumn{6}{|l|}{ FRANCÉS } \\
\hline LIC. EN ARTES & $1,21 \%$ & $23,59 \%$ & $6,77 \%$ & $2,03 \%$ & $8,40 \%$ \\
\hline \multicolumn{6}{|l|}{ VISUALES } \\
\hline LIC, LENGUA & $10,64 \%$ & $13,30 \%$ & $-0,82 \%$ & $15,81 \%$ & $9,73 \%$ \\
\hline \multicolumn{6}{|l|}{ CASTELLANA E } \\
\hline \multicolumn{6}{|l|}{ INGLÉS } \\
\hline LIC, CIENCIAS & $6,96 \%$ & $15,82 \%$ & $2,24 \%$ & $13,86 \%$ & $9,72 \%$ \\
\hline \multicolumn{6}{|l|}{ NATURALES Y } \\
\hline \multicolumn{6}{|l|}{ EDUCACIÓN } \\
\hline \multicolumn{6}{|l|}{ AMBIENTAL } \\
\hline LIC. EN CIENCIAS & $7,96 \%$ & $11,85 \%$ & $1,96 \%$ & $14,21 \%$ & $8,99 \%$ \\
\hline \multicolumn{6}{|l|}{ SOCIALES } \\
\hline LIC. EN FILOSOFÍA Y & $8,33 \%$ & $15,96 \%$ & $5,49 \%$ & $17,29 \%$ & $11,77 \%$ \\
\hline \multicolumn{6}{|l|}{ LETRAS } \\
\hline LIC. EN INFORMÁTICA & $4,31 \%$ & $18,61 \%$ & $-2,94 \%$ & $14,13 \%$ & $8,53 \%$ \\
\hline
\end{tabular}




\begin{tabular}{lccccc} 
LIC. EN LENGUA & $9,26 \%$ & $15,91 \%$ & $4,32 \%$ & $17,85 \%$ & $11,83 \%$ \\
$\begin{array}{l}\text { CASTELLANA Y } \\
\text { LITERATURA }\end{array}$ & & & & \\
$\begin{array}{l}\text { LIC. EN } \\
\text { MATEMÁTICAS }\end{array}$ & $-3,43 \%$ & $7,69 \%$ & $-4,30 \%$ & $14,44 \%$ & $3,60 \%$ \\
MEDICINA & $9,90 \%$ & $13,77 \%$ & $4,50 \%$ & $7,36 \%$ & $8,88 \%$ \\
MEDICINA & $3,34 \%$ & $6,95 \%$ & $-0,03 \%$ & $9,95 \%$ & $5,05 \%$ \\
VETERINARIA & $5,97 \%$ & $16,09 \%$ & $2,31 \%$ & $10,65 \%$ & $8,76 \%$ \\
PSICOLOGÍA & $14,85 \%$ & $9,66 \%$ & $9,16 \%$ & $16,53 \%$ & $12,55 \%$ \\
QUÍMICA & $6,24 \%$ & $22,60 \%$ & $0,07 \%$ & $9,67 \%$ & $9,65 \%$ \\
SOCIOLOGÍA & $2,18 \%$ & $15,37 \%$ & $6,70 \%$ & $8,32 \%$ & $8,14 \%$ \\
ZOOTECNIA & & & & & \\
\hline
\end{tabular}

Fuente: esta Investigación

Razonamiento cuantitativo: El área de razonamiento cuantitativo es la que presenta un mayor valor agregado en relación al promedio de los resultados estadísticos por programa académico (13,4\%). Los programas de Licenciatura en Artes Visuales, Sociología y Diseño Gráfico resaltan con el resultado en porcentaje de valor agregado en este módulo teniendo en cuenta que de acuerdo a los planes de estudio que la Universidad de Nariño ofrece para estas carreras, el componente matemático es moderado en comparación con otras carreras, como ingenierías o las del área económica y administrativa; a su vez, el valor agregado obtenido por el programa de Artes Visuales, es el mayor resultado registrado en los datos obtenidos entre los módulos. En contraste, programas como Licenciatura en Matemáticas, Ingeniería Civil e Ingeniería electrónica resultan en un bajo valor agregado en este módulo, lo que resulta interesante si se tiene en cuenta que los planes de estudios de estas carreras, dada su naturaleza, poseen un fuerte componente matemático.

Lectura crítica: El promedio por programas de valor agregado obtenido en el módulo de Lectura Crítica es de 5,72\%, con un grado de dispersión de los datos de cinco puntos porcentuales. En la Universidad de Nariño existen módulos obligatorios para todos los estudiantes que ingresan a primer semestre de cualquier programa ofertado en la institución, denominado "Lectura y producción de textos", los cuales van encaminados a fortalecer las 
aptitudes en el área de la lectoescritura que los estudiantes traen de su educación media, y que a su vez, le contribuye al manejo de los temas de aprendizaje en el desarrollo de su plan de estudios.

Esto sumado a la práctica de estas aptitudes en cada campo con la elaboración de trabajos e investigaciones, explica en gran medida el resultado del valor agregado en carreras como Ingeniería en Producción Acuícola, Química, Licenciatura en Lengua Castellana y Medicina. Por otra parte, el resultado negativo para carreras como la Ingeniería Civil, Licenciatura en Matemáticas, Ingeniería Electrónica y Física, se pude explicar a la naturaleza práctica y experimental de las mismas.

Competencias ciudadanas: En promedio, el resultado para los programas fue de 2,76\%, siendo relativamente bajo comparado con los obtenidos en las otras áreas estudiadas. Se destacan programas como Química, Ingeniería en Producción Acuícola y Comercio Internacional con los mejores resultados en este módulo. Por otra parte, programas como Ingeniería Civil, Licenciatura en matemáticas y Física tienen un menor valor agregado en la formación ciudadana de sus estudiantes. Es interesante el resultado obtenido para el programa de Economía, siendo esta una ciencia social; de lo que se sugiere un mayor seguimiento a este y otros programas en el desarrollo de competencias genéricas.

Inglés: La formación en lengua extranjera, particularmente en inglés, es una necesidad fuerte para los profesionales formados en la Universidad de Nariño; por tanto, esta institución cuenta con el Centro de Idiomas, donde se ofrece la formación en diferentes lenguas. Para los programas académicos, los planes de estudios tienen como mínimo dos niveles de inglés, y la formación puede extenderse hasta los seis niveles que se ofrecen en la universidad. Además, se debe tener en cuenta que la gran mayoría de la producción bibliográfica en todos los ámbitos de la investigación se hace en idioma inglés.

A partir de esto se justifica los resultados agregados por el curso de un plan de estudios en la Universidad de Nariño en relación a las pruebas de inglés. El resultado promedio es de 11,95\% con una desviación de cuatro puntos porcentuales. En este caso, todos los programas agregaron valor en la formación de esta área, resaltando los programas de Licenciatura en Lengua 
Castellana y Literatura, Licenciatura en Filosofía y Letras y Química. Los programas con el menor resultado obtenido son Zootecnia, Medicina, Física, Licenciatura en Artes Visuales e Ingeniería Electrónica.

De un análisis promedio entre los resultados obtenidos en las cuatro pruebas evaluadas, el programa que más otorgó valor en las pruebas genéricas fue Ingeniería en Producción Acuícola, seguido de Química y el programa de licenciatura en Lengua Castellana y Literatura. Por otra parte, los programas que menos valor agregado otorgaron desde el curso de su plan de estudios a los módulos evaluados en las pruebas genéricas fueron Ingeniería Electrónica, Física e Ingeniería Civil. Los resultados se muestran en la Tabla 7.

\section{Tabla 7}

Mayores y menores porcentajes de valor agregado promedio. Universidad de Nariño 20102014

\begin{tabular}{clc}
\hline \multicolumn{1}{c}{ Escala } & \multicolumn{1}{c}{ Programa } & Resultado \\
\hline Mayores & Ing. En Producción Acuícola & $13,03 \%$ \\
& Química & $12,55 \%$ \\
& Lic. En Lengua Castellana Y Literatura & $11,83 \%$ \\
& Lic. En Filosofía Y Letras & $11,77 \%$ \\
& Comercio Internacional Y Mercadeo & $11,13 \%$ \\
& Medicina Veterinaria & $5,05 \%$ \\
& Lic. En Matemáticas & $3,60 \%$ \\
& Ing. Civil & $3,09 \%$ \\
& Física & $1,08 \%$ \\
& Ing. Electrónica & $-1,44 \%$ \\
\hline
\end{tabular}

Fuente: esta Investigación

La educación tiene un papel importante en el desarrollo económico y social de los países en general y de las regiones en particular, por lo cual contribuir a mejorar el escenario desde la academia y tener la posibilidad de medir dicho papel con respecto a pruebas genéricas se torna de vital importancia. Los procesos educativos tienen una consecuencia directa en diferentes 
esferas de la vida del individuo y de la sociedad, además de que implican tanto una inversión importante en el gasto público, como en los desembolsos individuales que hace cada uno para formarse y obtener una educación de calidad.

En aras de adquirir nuevos conocimientos, se busca que las instituciones educativas tengan un impacto directo en el crecimiento personal de los contenidos y habilidades de los individuos, permitiendo visualizar un valor agregado que se manifieste en un amplio conocimiento sobre especialidades elegidas por las personas y en el desempeño de sus habilidades en relación a las líneas de acción en las que se desempeñe laboralmente. Dicho de otra manera, las personas buscan instituciones que les aseguren un aporte a los conocimientos adquiridos y que sea reflejada en procesos evaluativos y autoevaluativos, presentes continuamente en el modelo educativo vigente.

\section{CONCLUSIONES}

- Uno de los elementos importantes para el desarrollo de las regiones es la educación superior de calidad, de ahí la importancia de mantener una valoración del aporte que generan las Instituciones de Educación superior IES a sus egresados lo que se ha denominado Valor Agregado, lo cual posibilita establecer políticas claras de mejoramiento profesional en un mundo globalizado.

- Las teorías del capital humano establecen a la educación y en especial a la educación superior como una inversión que incrementa la productividad en el largo plazo y por ende la remuneración fruto de la misma, lo cual genera un círculo virtuoso de mejoramiento en la calidad de vida de la sociedad.

- El aporte de la Universidad de Nariño a las competencias genéricas (Lectura crítica, Razonamiento cuantitativo, Competencias ciudadanas, Comunicación escrita e Inglés) de sus estudiantes a través de las pruebas Saber 11 y Saber Pro, se pudo establecer para el periodo 2010-2014, esto dadas las condiciones de estructuras en las pruebas Saber 11 y Saber Pro comparables según metodología del ICFES. 
- Durante el periodo estudiado la Universidad de Nariño genera en promedio un $10 \%$ de valor agregado a sus estudiantes, siendo el módulo de Inglés el que más aporta, ubicándose en un 13,50\%, seguido de Razonamiento cuantitativo con 13,37\%. El componente en el que menos le aportó la Universidad a los estudiantes es Competencias ciudadanas con un 2,89\%. En la competencia de Razonamiento cuantitativo se hace posible observar que se obtuvo un máximo de $40 \%$ en valor agregado generado por la universidad, mientras que en los mínimos, el que más se resalta es el obtenido en Inglés con un -35\%.

- Un análisis del valor agregado en el periodo determina que hay grandes fluctuaciones de este en dicho periodo, con una tendencia ascendente que evidencia que hay un mejoramiento en el desempeño de las competencias genéricas.

- En torno al análisis del valor agregado por programas de la Universidad (33), se puede concluir que los mayores puntajes de valor agregado se obtienen en los módulos de Razonamiento cuantitativo e Inglés, y los módulos con menores porcentajes de valor agregado son Lectura crítica y Competencias ciudadanas. En estos últimos hay algunos programas (8) que arrojan datos negativos de valor agregado. Así mismo, se puede establecer que casi todos los programas (32 de los 33), presentan un total de valor agregado positivo para el periodo analizado, solo un programa presenta un total de valor agregado negativo, Ingeniería electrónica.

- De los programas con mayor valor agregado total para el periodo 2010-2014 están tres: Ingeniería en Producción Acuícola con 13,03\%, Química con 12,55\% y Licenciatura en Lengua Castellana y Literatura con 11,83\%. Los tres programas con el valor agregado total más bajos son: Ingeniería electrónica con -1, 44\%, Física con 1,08\% y Licenciatura en Matemáticas con el 3,60\%. 


\section{REFERENCIAS}

(1) Banco Mundial. (2018). Gasto público en educación, total (\% del PIB). Recuperado el 2018, de World Bank Group: https://datos.bancomundial.org/indicador/SE.XPD.TOTL.GD.ZS

(2) Cardona, M., Montes, I., Vásquez, J. J., Villegas, M., \& Brito, T. (Abril de 2007). Capital Humano: Una mirada desde la educación y la experiencia laboral. Obtenido de Semillero de Investigación en Economía de EAFIT -SIEDE- Grupo de Estudios Sectoriales y Territoriales -ESyT-.

(3) Clavijo, G. (2008). La evaluación del proceso de Formación. Cartagena de Indias.

(4) Instituto Colombiano para el Fomento de la Educación Superior. (Diciembre de 2013). Sistema Nacional de Evaluación Estandarizada de la Educación, Alineación del examen SABER 11². Recuperado el 2018, de ICFES.

(5) Instituto Colombiano para el Fomento de la Educación Superior. (2014). Recuperado el 2018, de ICFES SABER PRO.

(6) Instituto Colombiano para el Fomento de la Educación Superior. (Febrero de 2014). El antes y el ahora del $\begin{array}{llllll}\text { examen. } & \text { Recuperado } & \text { el Julio }\end{array}$ http://www.icfes.gov.co/index.php?option=com_k2\&view=item\&layout=item\&id=520\&Itemid=344

(7) Instituto Colombiano para el Fomento de la Educación Superior. (Agosto de 2014). Medición de los efectos de la educación superior en Colombia sobre el aprendizaje estudiantil. Bogotá D.C., Colombia.

(8) Instituto Colombiano para el Fomento de la Educación Superior. (Septiembre de 2015). Información de la prueba Saber Pro. Recuperado el 2018, de http://www2.icfes.gov.co/instituciones-educativas-ysecretarias/saber-pro/informacion-de-la-prueba-saber-pro

(9) Instituto Colombiano para el Fomento de la Educación Superior. (2017). Información general del examen Saber Pro. Recuperado el 2018, de ICFES: http://www.icfes.gov.co/estudiantes-y-padres/saber-proestudiantes/informacion-general-del-examen

(10) Instituto Colombiano para el Fomento de la Educación Superior. (2018). Quienes somos. Obtenido de http://www.icfes.gov.co/quienes-somos

(11) Instituto Colombiano para el Fomento de la Educación Superior. (2018). Valor agregado y Aporte relativo. Obtenido de http://www.icfes.gov.co/investigadores-y-estudiantes-posgrado/valor-agregado-aporte-relativo 
Cálculo del valor agregado generado por la Universidad de Nariño en relación a las pruebas saber 11 - saber pro $2010-2014$.

BURGOS FLÓREZ _ Marco Antonio, RUALES SUÁREZ _ Katherin Julieth, BASTIDAS GARCÍA _ Yhony Estivel, ORTIZ

(12) Isáziga, C., \& Gabalá, J. (Mayo de 2014). La intervención académica en la construcción de una sociedad con calidad. Aproximación a través del análisis del valor agregado en el difernecial SABER11-SABER PRO por regiones CORPES. Estudios sobre calidad de la educación en Colombia. Bogotá D.C., Colombia.

(13) Ministerio de Educación Nacional. (2011). Propuesta de lineamientos para la formación por competencias en educación superior. Bogotá D.C.

(14) Ministerio de Educación Nacional. (2018). MINISTERIO DE EDUCACION NACIONAL. ¿Qué es la Evaluación de Competencias? Obtenido de http://www.mineducacion.gov.co/proyectos/1737/article210839.html

(15) Monroy, A., Aguirre, C., \& Espitia, A. (2018). Propuesta metodológica para identificar el valor agregado de programas de ingeniería a partir del análisis de resultados de pruebas estandarizadas. Recuperado el 2018, de Asociación Colombiana de Facultades de Ingeniería. Revista Educación en Ingeniería. 13(25), pp. 102-107.

(16) Sen, A. (Diciembre de 1997). Capital Humano y Capacidad Humana. Recuperado el Enero de 2019 